\title{
DOÑANA Y SU ENTORNO: CONSERVACIÓN Y EXPLOTACIÓN
}

\author{
José Manuel RUBIO RECIO *
}

Tanto río de tinta sobre Doñana y tantas palabras, noticias, pareceres, opiniones y declaraciones de gentes de las más diversa condición, no están haciendo sino sembrar confusión. Y no digamos nada de la presencia de gobernantes y políticos, -alguna sospechosamente asidua- que se arroban y extasian en el secarral o humedal doñanero, sobre todo si está la televisión o la prensa delante. Doñana para esas personas es sólo fuente de imagen, porque Doñana, como muy acertadamente expresó Aquilino Duque al titular su libro sobre el Parque Nacional que se asienta sobre dicho lugar, es un mito y como tal se explota. En el fondo, no se si será una manifestación de "si aquí se invitaba a los Reyes de España y a otras gentes de la nobleza, en otras épocas, -denostadas, eso sí-, ¿por qué no voy a venir yo?-".

Mito que, por otra parte, juega entorno a una realidad biológica muy valiosa, no excesivamente espectacular, en un paisaje de amplios horizontes, poco o nada original, salvo la conspicuidad del área de las dunas. Por más que se la quiera magnificar, Doñana carece de belleza escénica. El que se la valore, -y mucho-, no nos debe hacer ignorar esa realidad.

Una serie de circunstancias naturales, y otras históricas y sociales, se combinaron para mantener este espacio relativamente poco intervenido por la mano del hombre.

Quizá convenga precisar alguna información sobre dicho espacio, para que al ser invocado como palanca del desarrollo de los municipios limítrofes del mismo, sepamos a qué atenernos.

Pero antes de nada hay una pregunta, aparentemente fácil de contestar, pero que para la gente, en general, creo que no lo sea: ¿A qué espacio se le llama Doñana? Comencemos por ello.

* Catedrático de Geografía Física. Universidad de Sevilla. 


\section{EL ESPACIO DOÑANERO}

Históricamente, el Coto de Doñana u Oñana era una finca de los Duques d Medina Sidonia, que cubría todo el espacio de las dunas y matorrales, entre e mar y la marisma, que va ganando en anchura hacia el Oeste, más una franja d€ marisma sensiblemente paralela a lo anterior, hasta la línea irregular que marca e cauce del arroyo de la Rocina en su camino hacia el Guadalquivir. Este cauce c "caño" es precisamente el límite municipal de Almonte, que históricamente, consideró que la apropiación de esa porción de marisma por los Duques de Medinz Sidonia fue una usurpación ilegal.

La Doñana tradicional era tan sólo esto y fue un espacio sobre el que los almonteños mantuvieron, con alternativas de acuerdo, revindicaciones de propiedad y de uso, frente a la casa ducal. (Vid. Ojeda Rivera, J.F. 1987).

El término municipal de Almonte tiene una extensión de nada menos que 85.914 Has., lo que le convierte en el mayor de la provincia de Huelva. Lá superficie de la Doñana tradicional era de unas 27.000 Has. de las que 6.000 Has eran las de las marismas reivindicadas por Almonte. El municipio que le sigue er extensión es el de Aroche, en plena Sierra Morena, que tiene 49.844 Has. que insistiendo, son de sierra.

Pero hablemos, antes de pasar a otras cuestiones, del conjunto de Las Marismas del Guadalquivir. De lo que eran, ya que en buena parte han dejado de serlo.

Su extensión era nada menos que de 136.000 Has. Y es precisamente ese espacio físico el causante de la existencia de un Doñana biológico.

Es la vida vinculada a esa superficie acuática la que tiene importancia y trascendencia biológica no sólo a nivel nacional, sino a nivel de dos regiones biogeográficas,. la Paleártica y la Paleotropical, que se extienden por todo e] conjunto de Africa, Europa y Asia al Norte del Himalaya.

El que además haya otros ecosistemas distintos contiguos a los marismeños: ecosistemas no acuáticos, como los de las dunas o los de los "cotos", no deja de ser algo circunstancial, si bien enriquezca mucho al conjunto total y le convierta en un complejo biótico más rico aún.

A costa de ello, lo que me interesa destacar es que el Parque Nacional de Doñana sólo se justifica y es viable en tatno en cuanto la marisma que aún se conserva, no vea más modificados substancialmente sus caracteres y su ciclc hidrológico. Ya que las alteraciones que ha venido sufriendo en las últimas décadas la han colocado en una situación tan al límite, que se puede afirmar que nos encontramos en el umbral de su aniquilación. 


\section{DOÑANA, PARQUE NACIONAL, SOBRE TODO ACUÁTICO}

Hay que tener en cuenta una realidad incuestionable: Doñana sin la marisma no tiene razón de ser. Es algo que hay que asumir como axioma, lo que supone que cualquier actuación que afecte a Doñana o a su entomo, en vez de tender a usos del agua que disminuyan las que por unos caminos u otros fluyen hacia la marisma o alimentan los acuíferos, debe buscar otras altemativas de aprovisionamiento. Incluso si fuera factible, se deberá proceder como parcialmente se hizo con el programa de regeneración hídrica a partir de las aguas del Guadiamar.

Si queremos que Doñana subsista habrá que seguir por la vía que se inició con las antedichas aguas, llevando las actuaciones a otras fluencias. Porque lo que sigue ocurriendo es justamente lo contrario. Se persiste en la utilización de los acuíferos, amparándose en los informes contradictorios sobre su riqueza y sobre su capacidad de recarga, aunque los que tengan más visos de ser reales sean Ios del Prof. Llamas, los elaborados por la misión WWF/UICN/ADENA al Parque Nacional de Doñana en 1988 (Hollis, T. 1989) o los del IGME. Precisamente estos últimos, realizados en 1987, fueron acompañados de unas simulaciones que arrojaron unos resultados desoladores para el Parque Nacional de Doñana en los años posteriores al principio del próximo siglo, en el que se producirían notables cambios ecológicos en extensas zonas, provocados en su totalidad por los descensos de los niveles de los acuíferos, que afectarían al más fundamental para la vida del Parque: el freático superior.

El aumento de población de la zona y de sus exigencias de agua, que por otra parte no dejan de ser lógicas, en el poblamiento tradicional, en el Rocío o en Matalascañas, hace crecer la extracción de agua, que sólo puede tomarse de los acuíferos. Por otro lado está la demanda de los agricultores, que ven en el regadío una mejora de su situación. Además, está el problema de las aguas que se vierten y que en su mayor parte van hacia la marisma; son aguas cuyo nivel de contaminación también va en aumento, ya que no sólo los particulares incumplen Ias normad de depuración o de no contaminación, sino que son las propias administraciones municipales las que no cumplen con la normativa y no vigilan para que así se haga.

Se está ante un dilema muy serio porque, insistiendo, cualquier cantidad de agua extraída o restada del entomo de Doñana o toda contaminanción de las que en él se vierten, es una agresión al Parque. En el Plan Director Territorial de Coordinación de Doñana y su entorno esto se dice, pero a renglón seguido se entra en contradicción porque también se dice que "en las marismas saneadas se facilitará y asegurará la disponibilidad de los recursos hídricos necesarios" y como no hay otras, tendrá que ser a costa de las vitales para el Parque. 
Es evidente que habrá que establecer prioridades. Y si la prioridad fuera, tal como yo creo que debiera de ser, Doñana, y con ello se perjudica al sector productivo, la solución hay que buscarla a través de la compensación económica. También habrá que convencerse que conservar cuesta dinero; y habrá que asumirlo. Porque el que Doñana se seque es un mal sin arreglo, por más dinero que le echemos al asunto.

Había empezado a hablar de Las Marismas y he derivado al problema del agua, quizá porque se trata de algo consustancial con ellas, pero es hora de analizar a quién pertenecen y en qué proporciones y en qué grado de transformación de hallan. Porque ¿Qué es lo que queda en esas 130.000 Has. de marisma semivirgen?

\section{EL DOMINIO MUNICIPAL DE LOS ESPACIOS DE DOÑANA Y EL PROCESO COLONIZADOR}

Administrativamente, la propiedad por municipios es la siguiente, con independencia de la posesión personal que haya en cada uno de ellos:

Puebla del Río (Sevilla)

Aznalcázar (Sevilla).

Lebrija (Sevilla)

Hinojos (Huelva)

Sanlúcar de Barrameda (Cádiz)

Utrera (Sevilla)

Las Cabezas de San Juan (Sevilla)

Almonte (Huelva)

Trebujena (Cádiz)

Dos Hermanas (Sevilla)

Los Palacios (Sevilla)
35.965 Has.

33.165 Has.

16.000 Has.

11.612 Has.

$11.000 \mathrm{Has}$.

7.I00 Has.

6.400 Has.

6.000 Has.

5.000 Has.

2.300 Has.

1.700 Has.

Total

136.242 Has.

De ellas, las de Lebrija, Trebujena, Sanlúcar de Barrameda, Utrera, Dos Hermanad, Los Palacios y Las Cabezas de San Juan corresponden a la margen izquierda del Guadalquivir, sumando $48.500 \mathrm{Has}$., con lo que a la margen derecha le corresponden 87.742 Has. Y, en principio, son estas segundas las marismas clave para el Parque Nacional de Doñana; las primeras, en algún caso, pueden formar parte de los espacios "colchón” que configuran el Preparque. 
Ahora, para comprender el grado de reducción a que han sido sometidas, haré un breve recorrido histórico del proceso colonizador, que enlazará, al avanzar el tiempo, con el de conservación.

El espacio Marismas del Guadalquivir, dunas de Doñana y cotos periféricos, pero sobre todo Las Marismas, era un vacío humano, por las limitaciones inherentes a los ritmos de inundación, la salinidad de los suelos y la presencia del paludismo como enfermedad endémica (Vid. Valverde, J.A. 1979). Aunque se esgrime como razón del vacío humano la existencia de gran propiedad, -y puede servir para algunas partes-, su inexistencia, como ocurre en las marismas de Puebla del Río, Aznalcázar o Hinojos, no facilitó el que dicho espacio fuera ocupado por el hombre y, de esa suerte, persistió durante siglo como reino de la avifauna propia de ella.

Al mismo tiempo, como todo espacio vacío, y en este caso acuático, suscitó, al igual que en toda Europa, después de la Edad Moderna, el interés de su saneamiento y posterior colonización. Así ocurrió, por ejemplo, con pantanos, lagunas y marismas italianas, cuya colonización tuvo carácter de paradigmática.

De esta suerte, desde el siglo pasado se suceden los intentos de conquista de espacios marismeños con fines agrícolas. (Vid. para más información o detalle Grande Covian, R., 1956, o González Arteaga, J. 1989, o Ojeda Rivera, J.F. 1987 y Zoido Naranjo, F. 1973). Durante años no se consiguieron logros substanciales ni estables y si tuviéramos que fijar una fecha en la que se comenzase a apreciar un cambio de signo de lo antedicho habría que llegar a años posteriores a la Guerra Civil de 1936.

Es sabido que en esa época, tanto en ámbitos gubernamentales nacionales como regionales, existió toda una política colonizadora y explotadora, un poco forzada por las circunstancias que se vivieron. Y nadie pensaba entonces en conservación de la Naturaleza fuera de ámbitos con la espectacularidad de las montañas alpinas o la conservación de alguna especia cinegética como la Capra hispánica.

El entonces Coto de Doñana era simplemente un espacio mítico, donde se daban cacerías para un corto número de personas allegadas a los propietarios y a las que años atrás había sido invitado asiduo el Rey Don Alfonso Xlll. Muy pocos sabían que desde mediado el siglo pasado el lugar había sido visitado con asiduidad por distintos grupos de científicos, -ingleses en su mayoría-, cuyas observaciones se publicaban en revistas de Biología y sus diarios se guardaron, a veces, en institutos de investigación, porque ya se consideraban de valor. En unas y en otros ya quedó patente la riqueza, sobre todo ornítica, de Las Marismas del Guadalquivir. 
También hay que decir que en ese mundo científico y en aquella época, no se insinuó la posibilidad o la necesidad de crear alguna figura de protección. Quizá no se pensaba que la riqueza de Doñana pudiera mermarse.

Los escasos naturalistas españoles de aquellos años nunca se ocuparon seriamente del área de Doñana, que más bien era, por la dificultad de acceso una "terra incognita".

La explotación del espacio Marismas-Doñana, en los años posteriores a la Guerra Civil del 36, se inicia en dos frentes: por un lado, con la polderización de las llamadas "islas" del Guadalquivir, que son los espacios que enmarcaban los entonces funcionales brazos en los que se abría el río a partir de Puebla: islas Mayor, Menor y Mínima. Los espacios marismeños de la margen izquierda habían sido saneados antes, por iniciativa de la Cía. Marismas del Guadalquivir, S.A. apoyándose en la Ley de 24 de Julio de 1918, referente al saneamiento de lagunas, marismas y terrenos pantanosos o encharcadizos. Y por otro, centrándose en los arenales y dunas costeras, más o menos estabilizadas, a base de una masiva repoblación forestal o sorprendentes ensayos de plantaciones como la del guayules, para la extracción del caucho. Los organismos encargados de operar en un campo y en otro fueron el Instituto Nacional de Colonización, -después IRYDA-, y el Patrimonio Forestal del Estado, respectivamente.

La actuación del primero se inició desde el margen Norte de Las Marismas, fundamentalmente sobre terrenos del término municipal de Puebla del Río (Sevilla), anulando la circulación del Guadalquivir por sus brazos, defendiendo de la inundación a las islas y encauzando la fluencia ocasional de las aguas del río Guadiamar. Era una actuación lejana al Coto de Doñana, pero que gravitaría sobre su espacio, el restarle aguas, aparte de reducir sensiblemente la extensión de la marisma. La actuación del segundo se desarrolló sobre todo en terrenos del municipio de Almonte y colisionó frontalmente con los intereses de los entonces propietarios del Coto de Doñana, cuando la repoblación quiso extenderse por los arenales y las dunas de aquella propiedad.

Gran parte del municipio de Almonte, -toda la que desde el "ruedo" llega hasta las dunas del Asperillo-, fue repoblada con pinos y con eucaliptos. Y el extenderla a propiedades privadas se justificó considerándola una actuación de “interés nacional".

Apoyándose en ello y por la resistencia a la introducción, sobre todo de eucaliptos, que manifestaban los propietarios de Doñana, intentando mantenerla tal cual, surgieron incluso propuestas de expropiación, que no llegaron a materializarse.

Se entabló una pelea jurídica y hubo que ceder a realizar alguna repoblación con ambas especies, tratando de primar al pino, con el que ya se había repoblado 
en Doñana en el siglo XVIII por los Duques de Medina Sidonia y había adquirido carta de naturaleza autorregenerándose.

\section{DEL INICIO DEL INTERÉS CIENTÍFICO, POR PARTE DE ESPAÑOLES, A LA CREACIÓN DEL PARQUE NACIONAL MIENTRAS SEGUÍA LA AGRARIZACIÓN}

Fue precisamente entonces -I952- cuando dos científicos españoles llegaron al Coto de Doñana y fueron gentilmente recibidos por la familia González Gordón, que eran unos de los propietarios, iniciándose una labor científica, propiciada por ellos y que, ampliándose poco a poco, llega a la situación actual.

Aquellos dos científicos, el uno todavía joven y el otro en plena madurez, eran los Profesores Valverde Gómez y Bernis Madrazo, a Ios que al año siguiente de su primera expedición tuve la suerte de acompañar.

Con esas visitas se inició una de las primeras campañas de anillado masivo de aves que se realizaba en nuestro país. Y ello fue posible, aparte del apoyo que recibimos de la familia González Gordon, por Ia acogida que la idea del anillamiento tuvo por parte del Museo de Ciencias Naturales de San Telmo y la Sociedad de Ciencias Naturales Aranzadi, ambos de San Sebastián, que facilitaron el material del anillado y los fondos para el desplazamiento y parte de la manutención de los expedicionarios.

Durante varios años, mientras la colonización de Las Marismas progresaba con los abundante medios del Estado, lo que se realizaba en el Coto de Doñana eran sólo unas campañas temporales de anillamiento e investigación que en algún momento sirvieron para argumentar en contra de las transformaciones repobladoras del Patrimonio Forestal del Estado.

Una beca de la fundación March concedida a J.A. Valverde para hacer un estudio en profundidad de Las Marismas y Doñana, se traduciría con el tiempo en la investigación base publicada sobre este espacio y sigue siendo de referencia obligada. (Valverde, J.A. 1960).

Entretanto, las expediciones científicas extranjeras se seguían sucediendo, pero hubo una que tuvo singular significación, por la relevancia de las personas que la componían y porque su Director recabó oficialmente del Consejo de Investigaciones Científicas que autorizase la presencia con ellos durante toda la campaña de José A. Valverde, que ya se hallaba vinculado a esa institución. Valga como muestra de las personas que componían aquella expedición la de Julián Huxley. De lo que se hizo hay una excelente referencia publicada (Mountfort, G. 
1958). Allí se cimentaron amistades que junto a las logradas en la Camarga francesa por J.A. Valverde desembocarían años después en la constitución de la WWF.

Ya se empezaba a hablar de una manera abierta y seria de la necesidad de proteger como fuera el espacio de Doñana, pero las aisladas voces hispanas sólo encontraban eco en el exterior.

El movimiento conservacionista europeo empezaba a dejarse sentir. Así, en 1962 se convocó la Conferencia M.A.R. a la que asistieron científicos de casi toda Europa y que desembocó en que tres años después se publicase la "Lista de las Zonas Húmedas de Europa y Norte de Africa de importancia internacional". En dicha lista se establecen dos categorías de zonas húmedas: las primeras -Aconsideradas de interés excepcional y las segundas -B- con menores volúmenes de avifauna pero con importancia internacional también. Pues bien, en España sólo hay cuatro de la primera categoría y Las Marismas del Guadalquivir ocupan el primer lugar. (Rubio Recio, J.M. 1977).

El que España estuviera representada y el que se publicase esa lista, con la preeminencia de nuestras Marismas del Guadalquivir, no supuso que se hiciera nada al respecto y, naturalmente los tecnócratas promotores de la colonización de las Marismas ignoraron lo dicho y siguieron en su tarea sin que mediase consideración alguna.

La falta de sensibilidad ante estos temas, manifestada entonces por nuestros políticos, no se verá muy mejorada con el paso del tiempo, como cualquier observador curioso y crítico puede apreciar sin mucho esfuerzo.

Bien es verdad que el fallo está también en las bases: en una falta de educación en relación con el respeto y la admiración que le debemos tener a la Naturaleza; y en su valoración como patrimonio a conservar.

Nuestros políticos quizá se vean presionados hacia la conservación por algunas minorías, pero sigue habiendo más mayorías de indiferentes, cuando no destructoras, y otras minorías desarrollistas a ultranza, que juegan demagógicamente y con facilidad con los indiferentes ofreciéndoles beneficios.

¿Cómo es posible que si de una Conferencia Internacional en la que participamos y en la que suscribimos una declaración sobre la importancia de un espacio, no hagamos nada, sino todo lo contrario, para su conservación?

Desde mi posición es algo que nunca entenderé, aunque parece ser una norma usual en nuestro mundo político. En la publicación mía, citada líneas atrás, en la que analizaba la significación de Doñana y las Marismas, terminaba con una frase que vuelvo aquí a repetir, ampliándola: "el solo hecho de que sea necesario argumentar, para defender al espacio en cuestión, es un índice desconsolador de irresponsabilidad científica, educacional y social, a nivel nacional”. 
En diversas ocaciones, en el ámbito de Doñana se han vendido fincas y el Estado no ha ejercido el legal derecho de retracto que tiene. Incluso cuando la compra, en un primer caso la realizó un súbdito extranjero y pese a que científicos españoles solicitasen que se ejerciese dicho derecho, porque se trataba de la finca que reúne mejores condiciones hidrológicas y siempre concentraba los mayores efectivos de aves acuáticas. Fue el caso de la finca llamada Las Nuevas, que pasó a las manos de Don Leo Biaggi para fines cinegéticos.

Quizá el que esto se produjera así actuó como fenómeno catalizador para que la protección de Doñana se iniciase por una vía para entonces muy sorprendente. La WWF, con fondos de diversas procedencias y de diversos países de Europa occidental, compra 6.700 Has. del Coto de Doñana y lo regalan, sin contrapartida alguna, al Consejo Superior de Investigaciones Científicas, para la constitución de una Reserva Biológica. (Vid. para conocer el proceso con detalle, p.e. Duque, A. 1977).

Creo que nunca se ha resaltado lo suficiente ni a los niveles que merece el valor de esa actuación, cuyo promotor hispano fue el Prof. Valverde, que actuó de fermento en la creación de la WWF, encontrando eco en las personas que constituyeron su primer Consejo de Dirección. Lo realizado con Doñana se convirtió con el tiempo en una práctica usual de la WWF. Lo que hay que destacar es que Doñana fue el primer caso.

Estábamos, cuando esto ocurría, en 1963. Pues bien, aún tendrían que pasar seis largos años para que las autoridades estatales españolas tomasen conciencia de que la Reserva Biológica creada era algo reducida y necesitaba cubrir un espacio mayor y se llegase a la creación del Parque Nacional de Doñana, elaborando una primera delimitación, que casi inmediatamente se detectó como insuficiente, faltando también espacios de Preparque.

El que esto se estuviera produciendo, en un clima en el que el tema conservación se había generalizado un tanto, no era obstáculo para que la colonización de Las Marismas diese un salto de gigante, surgiendo nuevas amenazas, porque el desarrollismo había abierto un nuevo frente.

El IRYDA había contactado, al iniciarse el Primer Plan de Desarnollo Nacional, con el organismo internacional FAO, al que involucró en el estudio y planificación de un ambicioso plan de agrarización de la parte Oeste de Las Marismas del Guadalquivir y sus terrenos limítrofes, pertenecientes en su mayoría al municipio de Almonte.

La FAO, con todas sus virtudes, que no se le regatean, no deja de ser un organismo desartollista, en cuyos técnicos, en principio, no tiene por qué pesar el factor conservación, y actúan en consecuencia. Son otros poderes los que tienen que ocuparse de eso. El recabar su colaboración, por parte del IRYDA, no dejó 
de ser una hábil maniobra para justificar con la presencia de una entidad de peso internacional reconocido, la continuación y ampliación de las actuaciones colonizadoras, e incluso conseguir ayudas y fondos cuantiosos. El día que salga a la luz el coste y la inversión de los proyectos y las instalaciones de los drenajes intubados de la marisma de Hinojos, por no poner más que un ejemplo, -intubación, por otra parte, de dudosa o inoperante funcionalidad a corto plazo-, sin coste para los usuarios, creo que dejará atónitos a técnicos y a expertos en política económica. Desde aquí les brindo el tema de investigación. En las zonas periféricas no marismeñas, lo que la FAO programó y la infraestructura también se creó, a fondo perdido, fue un área de regadíos intensivos, a base de la explotación de los acuíferos subyacentes. Ese fue el boceto del megalómano Plan Almonte Marismas, que bien merecería hoy un análisis desapasionado pero crítico.

En aquellos años, frente a la operatividad política y administrativa ingenieril del IRYDA, acaudillada por F. Grande Covián, sólo se oponían los escasos y no tan operativos, pero si luchadores, investigadores del Consejo Superior de lnvestigaciones Científicas, que iniciaban sus tareas como tales, pero que se veían involucrados en la defensa de un espacio que se veía agredido en multitud de frentes y que no estaba administrativamente consolidado.

\section{SE AGRAVA EL PROBLEMA DEL USO Y MANEJO DEL AGUA}

El avance de colonización de Las Marismas, por la margen derecha del encauzado caño del Guadiamar, impidiendo que las aguas del mismo siguieran su curso normal hacia el Oeste, le hizo decir entonces al Prof. Valverde que el Proyecto Almonte Marismas ponía en peligro los "lucios" de Mari López y el Lobo y que ni la finca de Las Nuevas ni Doñana se salvarían si desecaban el corazón marismeño.

Las exigencias de la conservación y la falta reconocida de viabilidad del Plan Almonte Marismas en su planteamiento original, al menos en buena parte, -otro organismo internacional, el Banco Mundial, desaconsejaba inversiones de la envergadura que requería el Plan en cuestión, por considerarlas de dudosa rentabilidad-, hicieron que se recortase el proyecto. Por otro lado, la presión conservadora conseguía, por fin, no sólo detener la desecación de las marismas del Oeste, sino lo que fue más importante, invertir el manejo del agua del citado río Guadiamar para que volviese a circular por los viejos cauces, abasteciendo a los "lucios" antes citados. Para la conservación de Doñana, la aprobación del llamado Plan de Regeneración Hídrica fue un verdadero logro. 
En el frente marismeño, por llamarle así por lo que tiene de lugar de confrontación, se llegó a un cierto "statu quo", con el que se salvaban algunas de las necesidades del Parque Nacional, pero ni mucho menos todas. La extracción de agua para el riego de la parte del Plan Almonte Marismas aprobado, del llamado acuífero 27 y los posibles vertidos de ella hacia la marisma, cargada de restos de abonos e insecticidas, son un conjunto de amenazas cuya solución no parece fácil, y de producirse, ha de inducir cambios substanciales en la marisma que se vea afectada por ellos.

El problema de la sobreexplotación del susodicho acuífero 27 por parte de las extraccciones de agua de riego, que no parece ser una cantidad grande, se liga con las que se hacen para las necesidades crecientes de la urbanización playera de Matalascañas, contigua al Parque Nacional, las del pueblo del Rocío, con su explosivo crecimiento y también contiguo al Parque; las de Almonte; y las de las posibles nuevas instalaciones turísticas costeras, que son las que han colmado el vaso de la polémica sobre el agotamiento o el cambio de nivel del acuífero.

\section{OPINIÓN Y COMENTARIO EN DEFENSA DEL PARQUE NACIONAL Y EN CONTRA DE DETERMINADOS POSICIONAMIENTOS}

Insistamos una vez más, la viabilidad del Parque Nacional de Doñana pasa por que pueda mantenerse un abastecimiento de agua, cuando menos, si no más, como el que hoy existe. Suficientemente ha sido agredido o disminuido el espacio marismeño, en el que incluso la superficie dedicada al arroz, que es el único aprovechamiento intensivo razonable, no produce a precios internacionalmente competitivos, tiene que ser subvencionada por el Estado y, como el mercado nacional está saturado, son los propios arroceros los que se oponen, a través de su sindicato, a posibles ampliaciones de las superficies dedicadas a este cultivo.

Tras de la constitución del Parque Nacional de Doñana, bajo sus límites quedan algo menos de 24.000 Has. de marismas. Ello supone tan sólo poco más del $16 \%$ del total de la extensión que tenían Las Marismas del Guadalquivir. El $16 \%$ del ámbito de lo que la conferencia M.A.R. había considerado espacio de importancia internacional. Curiosamente, todavía hay a quien le parece que Ia creación del Parque fue un lujo de utilidad política (Ojeda Rivera, J.F., 1987).

Puestos a hacer calificaciones en ese tono, no se lo que se podría decir de urbanizaciones de temporada y sin ninguna otra funcionalidad, como Matalascañas; o del montaje urbanístico entorno al fenómeno Rocío y lo que cuesta el alquiler de una casa en dicha aldea para una semana.

Bien, la constitución del Parque Nacional de Doñana supuso la génesis de una 
legislación cada vez más copiosa, que desembocó en la elaboración de un Plan Director Territorial de Coordinación de Doñana y su Entorno (Consejería de Obras Públicas y Transporte, 1989) que salvaguardase la integridad del Parque, creando áreas del Preparque, modificando alguno de los límites y generando una normativa que compatibilizase la vida y la supervivencia del Parque, con el desenvolvimiento de los municipios y sus gentes del entorno.

Ese entorno municipal liderado, quiérase o no reconocerlo, por el de Almonte, líder de todas las revindicaciones, exige compensaciones por lo que considera usurpación de unos teremos municipales. Y con ese planteamiento se inicia toda una dinámica de vindicaciones carentes de lógica y que llegan a absurdos inconcebibles.

Quien las plantea es Almonte, cuyo término municipal de nada menos de 86.000 Has., es, con mucho, el más extenso de la provincia de Huelva. Le sigue, en plena Sierra de Aracena, el de Aroche, con poco menos de 50.000 Has. Con las dimensiones de Almonte yo no tendría inconveniente en considerarle como un verdadero latifundio. Un gigantesto latifundio municipal.

Y si nunca se ha visto inconveniente en la expropiación de latifundios, cuando pertenecían a una persona, no veo por qué, ya que sólo se va a hacer con una parte, no se podría realizar aquí. Máxime si se hace en aras de una buena causa. ¿Por qué quejarse de la expropiación de una parte de un municipio para la creación o el mantenimiento de un Parque Nacional? Y si se considera que la expropiación debe de ser pagada, páguese. Tanto me da que se haga con un Duque, que con un señor cualquiera, que con un municipio, porque no se me alcanza que, ante un interés nacional, un municipio tenga que tener unos privilegios que se le niegan a otros.

La verdad es que la reacción normal debería ser la de sentirse orgullosos de la posesión de un bien natural de importancia reconocida a los niveles más altos y no estar esgrimiendo sistemáticamente su existencia como un despojo hecho a los municipios y como argumento palanca para recabar toda clase de prebendas y ayudas. Bastantes han conseguido con la sobredimensionalización del Rocío y Matalascañas; o con los beneficios de la repoblación forestal realizada por el Estado en bastante más de un tercio del término municipal; o con las concesiones de explotación del Centro de Acogida del Parque en El Acebuche y de la Cooperativa de visitas al Parque. ¿Con qué dinero se han montado los regadíos del Plan Almonte Marismas? ¿Van los almonteños a amortizar la inversión que allí se ha hecho? ¿No es cierto que el susodicho Plan es, en su mayor parte, un fracaso?

Aunque la extensión municipal de Almonte perdiera la ocupada por el Parque Nacional seguiría siendo el municipio más extenso de la provincia de Huelva. ¿Qué privilegio tiene Almonte frente a los municipios, por ejemplo, de la Sierra 
de Huelva, para que a él se le concedan prebendas y a los otros no? Por es camino va a haber que considerarle un aristócrata municipal.

Pero el ejemplo de la actitud de Almonte cunde y arrastra no sólo a los pueblos afectados por el PDTC de Doñana, sino a los políticos de turno. Así como muestra, voy a espigar algunos comunicados aparecidos en la prensa sevillana, que muestran las situaciones aberrantes a las que se está llegando y para las que ha servido de desencadenante el informe que sobre el Parque y su entorno ha emitido una comisión de expertos nombrada por el Presidente de la Junta de Andalucía. Al parecer, el PDTC de Doñana y su entorno, elaborado por los propios organismos competentes de la Junta hace menos de dos años no sirve.

El susodicho informe, que no aporta novedades dignas de mención, aconseja la organización para la zona de un desarrollo "blando", lo que deja de ser normal y correcto, porque nadie trata de que se haga un conservadurismo bloqueante de la vida del área.

Pues bien, de pronto, el Comité Ejecutivo de la Federación Onubense de Empresarios y las personas que ellos nominan "inversores europeos", -sin más precisiones-, dicen que lo propuesto por el informe de los expertos "arruina la provincia". Seamos serios: el espacio afectado por el PDTC de Doñana y su entorno, al que asombrosamente empieza a llamársele Comarca de Doñana, por más que lo estiremos no llega ni al $0,75 \%$ de la extensión provincial de Huelva y la mitad de él, además, pertenece a otras provincias. O sea que, al parecer, toda la economía de Huelva gravita sobre ese $0,375 \%$ de su extensión territorial. Sin comentarios.

Con motivo de la Expo'92 ha venido el Presidente de la C.E., Sr. J. Delors, que se ha entrevistado con el Presidente Chaves de la Autonomía andaluza que, quizá contagiado del pedigüeñismo de Almonte, ha solicitado formalmente del Sr. Delors, aparte de los normales fondos Feder que concede la C.E., la bonita cantidad de 50.000 millones de pesetas para el desarrollo de la comarca de Doñana. Mientras, el resto de los municipios de Huelva, como no tienen un Doñana, que sigan como están.

La lógica que se desarrolla -y que la prensa ni analiza ni critica-, no es de recibo, ni es siquiera lógica. Aunque es muy bonito decir que conservación y desarrollo son compatibles, la compatibilidad pasa por la necesidad de que el desarrollo no lesione, o lo haga mínimamente, a lo que se pretende conservar. Y en el caso que nos ocupa Doñana está archilesionada.

Me parece triste que no haya el suficiente sentimiento colectivo para que la corriente que existe de actuar en contra o a costa del Parque Nacional de Doñana, polarizando actuaciones e inversiones en su entorno, no se invierta y todo lo que se propone no se haga $30 \mathrm{Kms}$. más allá del límite del Parque, en aras de algo 
que todos deberíamos querer: potenciar el Parque Nacional de Doñana como patrimonio regional, nacional y mundial, con conciencia y orgullo por la suerte de poseer algo único a lo que mimar como una joya que, si la rompemos, jamás podremos recomponer.

\section{BIBLIOGRAFÍA CITADA}

CONSEJERÍA DE OBRAS PÚBLICAS Y TRANSPORTE (1989). Dirección General de Urbanismo. Junta de Andalucía. "Plan Director Territorial de Coordinación de Doñana y su Entorno", Sevilla, 305 págs. + mapas.

DUQUE, A. (1977). "El mito de Doñana". Servicio de Publicaciones del Ministerio de Educación. Madrid, 212 págs.

GONZÁLEZ ARTEAGA, J. (1989). "Las Marismas del Guadalquivir; etapas de un aprovechamiento económico". Tesis Doctoral (inédita). Universidad de Sevilla. Departamento de Geografía. Dos tomos más apéndices, 1.047 págs.

GRANDE COVIÁN, R. (1956). "Los suelos salinos. Su rescate y aplicación a las Marismas del Guadalquivir". Ministerio de Agricultura. Madrid, 173 págs.

HOLLIS, T. et al. (1989). "Las consecuencias de la extracción de aguas subterráneas para el futuro a largo plazo del Parque Nacional de Doñana". Misión WWF/UICN/ADENA al P.N. de Doñana, 87 págs., mimeografiadas.

MOUNTFORT, G. (1958). "Portrait of a Wilderness. The Story of the Coto Doñana Expeditions". Ed. Hutchinson. Londres, 240 págs.

OJEDA RIVERA, J.F. (1987). "Organización del territorio en Doñana y su entorno próximo (Almonte). Siglos XVIII-XX". Monografías de ICONA n. ${ }^{\circ} 45$, Madrid, 459 págs.

RUBIO RECIO, J.M. (1977). "Nota sobre la significación biogeográfica y los problemas de las Marismas del Guadalquivir y su Parque Nacional". Rev. Cuademos de Geografía de la Universidad de Granada, n. ำ 7, págs. 277-292.

VALVERDE, J.A. (1960). "Vertebrados de las Marismas del Guadalquivir. (Introducción a su estudio ecológico)". Rev. Archivos del Instituto de Aclimatación. Almería. Vol. IX, págs. 1-168. CSIC.

VALVERDE, J.A. (1979). "Coto Doñana". Ed. Olivo. Sevilla, 129 págs.

ZOIDO NARANJO, F. (1973). "Isla Mínima. Aspectos geográficos agrarios del arrozal sevillano". Servicio de Publicaciones de Ia Universidad de Sevilla, 155 págs. 